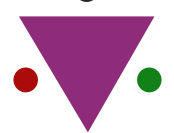

IJCRR

Section: Healthcare

Sci. Journal Impact

Factor: 6.1 (2018)

ICV: 90.90 (2018)

(c) (i) (3)

Copyright@IJCRR

\title{
Assessment of Association between Poor Oral Hygiene and Oral Cancer
}

\section{Harrita S1, Mahathi Neralla², Senthil Murugan $\mathbf{P}^{3}$}

\begin{abstract}
'Saveetha Dental College and Hospitals, Saveetha Institute of Medical and Technical Sciences, Saveetha University, Chennai, India; ${ }^{2}$ Reader, Department of Oral and Maxillofacial Surgery, Saveetha Dental College and Hospitals, Saveetha Institute of Medical and Technical Sciences, Saveetha University, Chennai, India; ${ }^{3}$ Associate professor, Department of Oral and Maxillofacial Surgery, Saveetha Dental College and Hospitals, Saveetha Institute of Medical and Technical Sciences, Saveetha University, Chennai, India.
\end{abstract}

\section{ABSTRACT}

Introduction: Oral cancer's incidence rates of oral cancer are higher in developed countries than in developing countries; however, in countries of Southern Asia, oral cancer is the most common cancer affecting males and the third most common one affecting females, after breast and cervical cancers. Poor oral hygiene is believed to play a role as a risk factor for head and neck cancer.

Objective: The aim of this study is to evaluate the relationship between poor oral hygiene and oral cancer.

Methods: In this retrospective study, a total of 86000 case sheets were reviewed, in which 477 case sheets of patients with poor oral hygiene were found. To act as a control, we retrieved 477 case sheets for each of the patients with good and fair oral hygiene.

Results: Among the 477 patients who had poor oral hygiene 32 patients were found to have oral cancer, for which the P-value was found to be statistically significant $(<0.05)$. The majority of the patients with poor oral hygiene who had oral cancer were males (27 patients) and P-value was found to be statistically significant $(<0.05)$. Among the 32 patients with oral cancer, 16 patients were in the age group of 36-55 years for which the P-value was found to be statistically significant $(<0.05)$.

Conclusion: Within the limits of the study, it is observed that there is a significant association between oral hygiene and oral cancer with males being the most affected gender and those in the age group of 36-55 years were found to be most affected. This shows the poor knowledge among patients about the maintenance of oral hygiene and the importance of regular dental check-up.

Key Words: Oral cancer, Poor oral hygiene, Oral hygiene index, Risk factors, Oral health, Oral hygiene maintenance

\section{INTRODUCTION}

Eighty-five percent of oral cancer occurs in the tongue, oropharynx, lips, and floor of the mouth. The tongue is the most common site for the occurrence of oral cancer. ${ }^{1}$ Squamous cell carcinoma accounts for over ninety percent of these tumors. ${ }^{2,3}$ Oral cancer occurs most commonly in middle-aged and older individuals but recently its occurrence has been seen in younger adults also. ${ }^{4-6}$ Tumors in the lips were more common in men but now have reduced significantly. Even though oral hygiene, septic and decayed teeth, and oral mucosal lesions such as leukoplakia have long been suspected to be risk factors for oral cancer, the role of such variables has been overshadowed for a long time by the results of studies relating tobacco smoking, alcohol drinking and, in Eastern countries, betel chewing to oral cancer risk. ${ }^{?}$
Although most studies have concentrated on smoking and alcohol consumption as the major risk factors, new investigations have focused on oral hygiene and diet. Exposure to important public health risks nutrition deficiency and oral health status, such as poor dentition, trauma due to ill-fitted dentures, either partially or totally, sharp or broken teeth, and frequency of poor oral hygiene have been suspected to be associated with oral tongue cancer. ${ }^{8}$

Oral hygiene is possibly associated with the risk of oral cancer, but the results are inconsistent. A previous study from Beijing showed that missing teeth and oral mucosal lesions increased the risk of oral cancer in women, but no association between the frequencies of brushing teeth, or wearing dentures were found. ${ }^{9}$ Balaram et al. reported an increased risk of oral cancer in women who brushed their teeth less

\section{Corresponding Author:}

Mahathi Neralla, Reader, Department of Oral and Maxillofacial Surgery, Saveetha Dental College and Hospitals, Saveetha Institute of Medical and Technical Sciences, Saveetha University, 162, PH Road, Chennai 600077, Tamil Nadu, India; Contact: +91 9840958142; Email: mahathin.sdc@saveetha.com

ISSN: 2231-2196 (Print)

Received: 10.09 .2020
ISSN: $0975-5241$ (Online)

Revised: 12.10 .2020
Accepted: 08.11 .2020
Published: 26.12.2020 
than once a day, and a meta-analysis showed that wearing dentures was a risk factor for oral cancer. ${ }^{10}$ Chang et al found regular visits to the dentist had a beneficial effect on the prevention of oral cancer. Vano et al. stated that oral health was correlated with education, and a high educational standard was associated with better oral hygiene.

Despite advances in surgery, radiation, and chemotherapy the five years' survival rate for oral cancer has not improved significantly over past several years and is still at 55\% percentage. There is a strong association between tobacco and oral cancer. chronic use of snuff has four times greater risk than other habits in developing into cancer. Alcohol consumption is in the moderate to high-risk category of developing into cancer. In India, high oral cancer rates are due to paan chewing. Poor oral hygiene is believed to play a role as a risk factor for head and neck cancer, especially for oral cancer. Only a few epidemiologic data exist about dental status and oral hygiene in patients. Thus the aim of this study is to evaluate the association between oral cancer and poor oral hygiene.

\section{MATERIAL AND METHODS}

\section{Study setting}

This study is conducted in a Private Dental College, Chennai, in a University set up. Approval from the ethical committee was obtained. Two examiners were involved in this study.

\section{Sampling}

This is a retrospective study in which a total of 86000 case sheets were reviewed, in which 477 case sheets of patients with poor oral hygiene patients were found. To act as a control we retrieved 477 case sheets for each of patients with good and fair oral hygiene. Oral hygiene status of these 1431 patients along with their case records was collected from the time period of June 2019 and March 2020. Cross verification of data for errors was done by the presence of additional reviewers and by photographs. Simple random sampling was done to minimize sampling bias. Data was entered in excel in a methodological manner and imported to SPSS. Incomplete data sheets were excluded from the study.

\section{Analysis}

IBM SPSS 23.0 software was used for statistical analysis. Independent variables included age, gender, poor oral hygiene. The dependent variable was oral cancer. Both descriptive and inferential statistics were done. Frequency distribution was done for age, gender, oral hygiene, and oral cancer. A comparison between groups was done using Chi-square test and $\mathrm{p}<0.05$ was considered as statistically significant.

\section{RESULT AND DISCUSSION}

Most of the patients with poor oral hygiene who had oral cancer were males (27 patients) (Figure 1) P-value was found to be statistically significant (0.0001) which is $<0.05$. Among these 1431 patients, 32 patients had oral cancer, for which the P-value was found to be statistically significant $(0.0001)$ which is $<0.05$ (Figure 2). There is a significant association between oral hygiene status and oral cancer. Among the 32 patients with oral cancer, 16 patients were in the age group of 36-55 years (Figure 3) for which the P-value was found to be statistically significant $(0.0001)$ which is $<0.05$. Thus, there is a significant association between age and oral cancer.

In this study, the majority of the patients who had oral cancer were males. This was like a study conducted by Silverman et al. ${ }^{1}$ Poor oral hygiene is one of the major risk factors for oral cancer. A study by Maier et al. states that the majority of patients with tumors seldom or never brush their teeth. ${ }^{11}$ Poor oral hygiene due to infrequent tooth brushing was associated with primary oral cancer in patients. ${ }^{12}$ Even though it is often hard to prove the real relation of cause to effect, one can no longer deny the detrimental role of tobacco, or of many alcoholic drinks, poor oral hygiene, nutritional deficiencies, short wave irradiation, and possibly certain viruses. These factors must further be investigated because the overall prognosis of oral cancer is poor. Late complications can be reduced by maintaining good oral hygiene. ${ }^{13}$ Previously our team had conducted numerous clinical trials, a review, and few surveys over the past five years. ${ }^{14-16}$ Now we are focusing on retrospective studies. The idea for the present study stemmed from the current interest in our community.

According to Madhu Laxmi M et al. study, the proto-oncogene c-MYC showed statistically significant upregulation in cancer tissue samples suggesting that OSCC in the south Indian population is not primarily affected by canonical Wnt signaling pathway. ${ }^{17}$ Sandeep B et al. has observed that in premalignant disorders like OSMF, buccal fat pad graft has proved to give better results as interposition material in surgical management. ${ }^{8}$ Though the present study is in accordance with previous literature, the limitations faced in the study were smaller sample sizes and the presence of a confounding factor.

\section{CONCLUSION}

Within the limits of the study, it is observed that there is a significant association between oral hygiene and oral cancer with males being the most affected gender and those in the age group of 36-55 years were found to be most commonly affected. This shows the poor knowledge among patients about the maintenance of oral hygiene and the importance of regular dental check-up. 


\section{Author Contribution}

All the authors contributed equally for the research

\section{ACKNOWLEDGEMENTS}

I would like to acknowledge Mr. Arun for the data collection.

\section{Conflict of Interests: Nil}

\section{REFERENCES}

1. Silverman S. American Cancer Society. Oral Cancer. PMPHUSA; 2003. pg. 212.

2. Neville BW, Damm DD, Allen CM. Odontogenic Cysts and Tumors.Diagn Surg Pathol Head Neck 2009; 785-838.

3. Jr JGR, Roy JG Jr. Silverman, Stanley J(oel) [Internet]. Oxford Music Online. 2002. Available from: http://dx.doi.org/10.1093/ gmo/9781561592630.article.o904725

4. Chen J, Katz RV, Krutchkoff DJ. Epidemiology of oral cancer in Connecticut, 1935 to 1985 . Cancer 1990;65:2796-802.

5. Llewellyn CD, Johnson NW. Risk factors for squamous cell carcinoma of the oral cavity in young people - a comprehensive literature review. Oral Oncology 2001;37: 401-18.

6. Schantz SP, Yu GP. Head and Neck Cancer Incidence Trends in Young Americans, 1973-1997, With a Special Analysis for Tongue Cancer. Arch Otolaryngol Head Neck Surg 2002;28: 268.

7. Olshan AF. Epidemiology, Pathogenesis, and Prevention of Head and Neck Cancer. Springer Science \& Business Media; 2010.pg.207.

8. Rezende CP de, de Rezende CP, Ramos MB, Daguíla CH, Dedivitis RA, Rapoport A. Oral Health Changes in Patients with Oral and Oropharyngeal Cancer. Braz J Otorhinolaryngol 2008;14:596-600.

9. Zheng T, Boyle P, Hu H, Duan J, Jiang P, Ma D, et al. Dentition, oral hygiene, and risk of oral cancer: a case-control study in Beijing, People's Republic of China. Cancer Causes Control 1990;1:235-41.

10. Balaram P, Sridhar H, Rajkumar T, Vaccarella S, Herrero R, Nandakumar A, et al. Oral cancer in southern India: The influence of smoking, drinking, paan-chewing and oral hygiene. Int J Cancer 2002;98:440-5.

11. Maier H, Zöller J, Herrmann A, Kreiss M, Heller WD. Dental Status and Oral Hygiene in Patients with Head and Neck Cancer. Otolaryngol Head Neck Surg 1993;108:655-61.

12. Jesudasan JS, Wahab PUA, Sekhar MRM. Effectiveness of $0.2 \%$ chlorhexidine gel and a eugenol-based paste on postoperative alveolar osteitis in patients having third molars extracted: a randomised controlled clinical trial. Br J Oral Maxillofac Surg. 2015 Nov 1;53(9):826-30.

13. Christabel A, Anantanarayanan P, Subash P, Soh CL, Ramanathan M, Muthusekhar MR, et al. Comparison of pterygomaxillary dysjunction with tuberosity separation in isolated Le Fort I osteotomies: a prospective, multi-centre, triple-blind, randomized controlled trial. Int J Oral Maxillofac Surg. 2016 Feb 1;45(2):180-5.

14. Rao TD, Kumar MP. Analgesic Efficacy of Paracetamol Vs Ketorolac after Dental Extractions. Res J Pharma Tech 2018;11(8):3375-9.

15. Mp SK. The emerging role of botulinum toxin in the treatment of orofacial disorders: Literature update. Asian J Pharm Clin Res 2017.

16. Packiri S, Gurunathan D. Management of paediatric oral ranula: a systematic review. J Clin 2017.

17. Marimuthu M, Andiappan M, Wahab A, Muthusekhar MR, Balakrishnan A, Shanmugam S. Canonical Wnt pathway gene expression and their clinical correlation in oral squamous cell carcinoma. Indian J Dent Res. 2018 May 1;29(3):291.

18. Patil SB, Durairaj D, Suresh Kumar G, Karthikeyan D, Pradeep D. Comparison of Extended Nasolabial Flap Versus Buccal Fat Pad Graft in the Surgical Management of Oral Submucous Fibrosis: A Prospective Pilot Study. J Maxillofac Oral Surg 2017; 16:312-21.

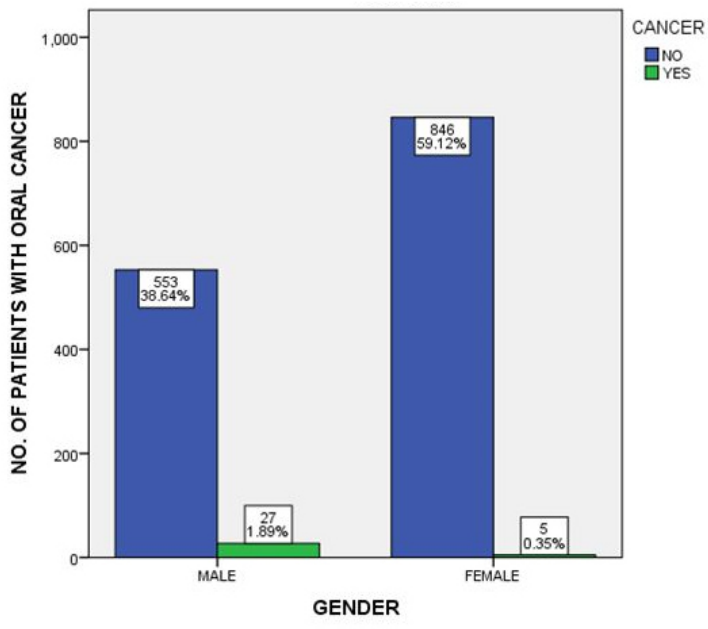

Figure 1: This is a bar graph showing the association between gender and oral cancer. The $\mathrm{X}$-axis denotes the gender and $\mathrm{Y}$ axis denotes the number of patients with oral cancer. Out of 1431 patients, 5 females (1.05\%) and 27 males (5.66\%) had oral cancer (green). The P-value was 0.000 (>0.05). Thus, there is a significant association between the gender of the patients and the presence of oral cancer. 


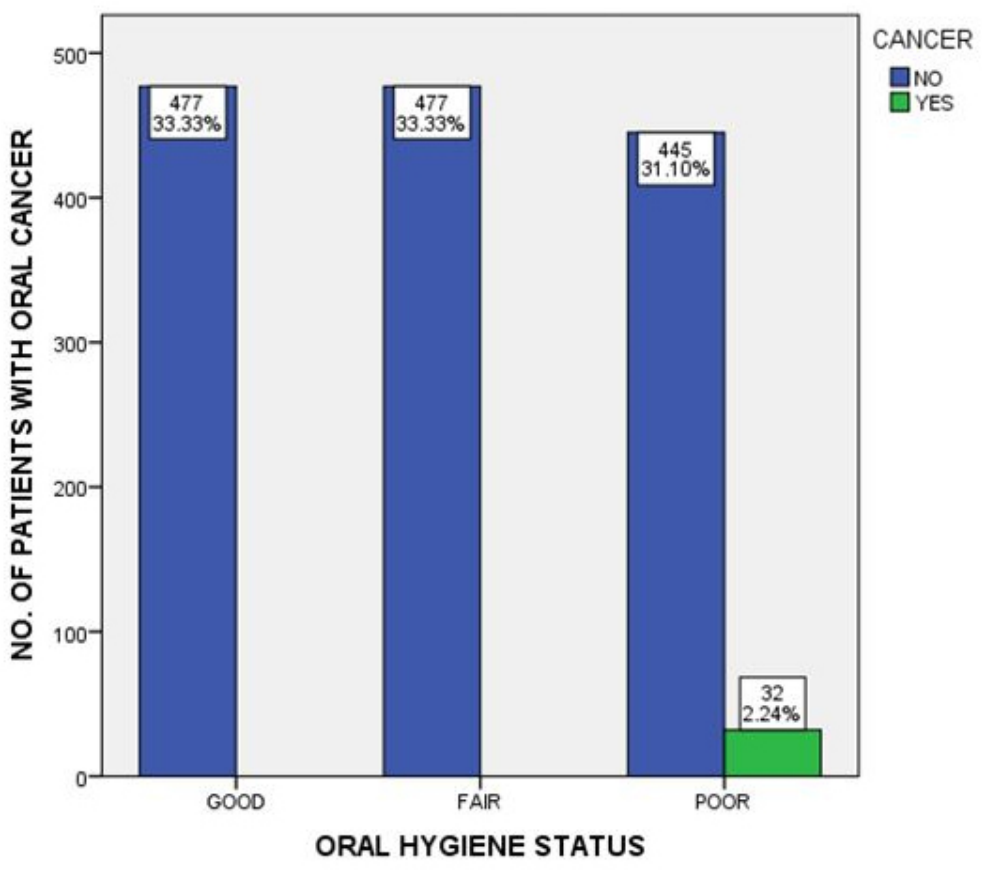

Figure 2: This is a bar graph showing the association between the oral hygiene status of the patients and oral cancer. The $X$ axis denotes the oral hygiene status of the patients and Y-axis denotes the number of patients with oral cancer. The green bar shows patients who had oral cancer and the blue graph shows patients who do not have oral cancer. Out of 1431 patients, 32 patients with poor oral hygiene $(2.24 \%)$ had oral cancer. The P-value was $0.000(<0.05)$. Thus, there is a significant association between oral hygiene status and oral cancer.

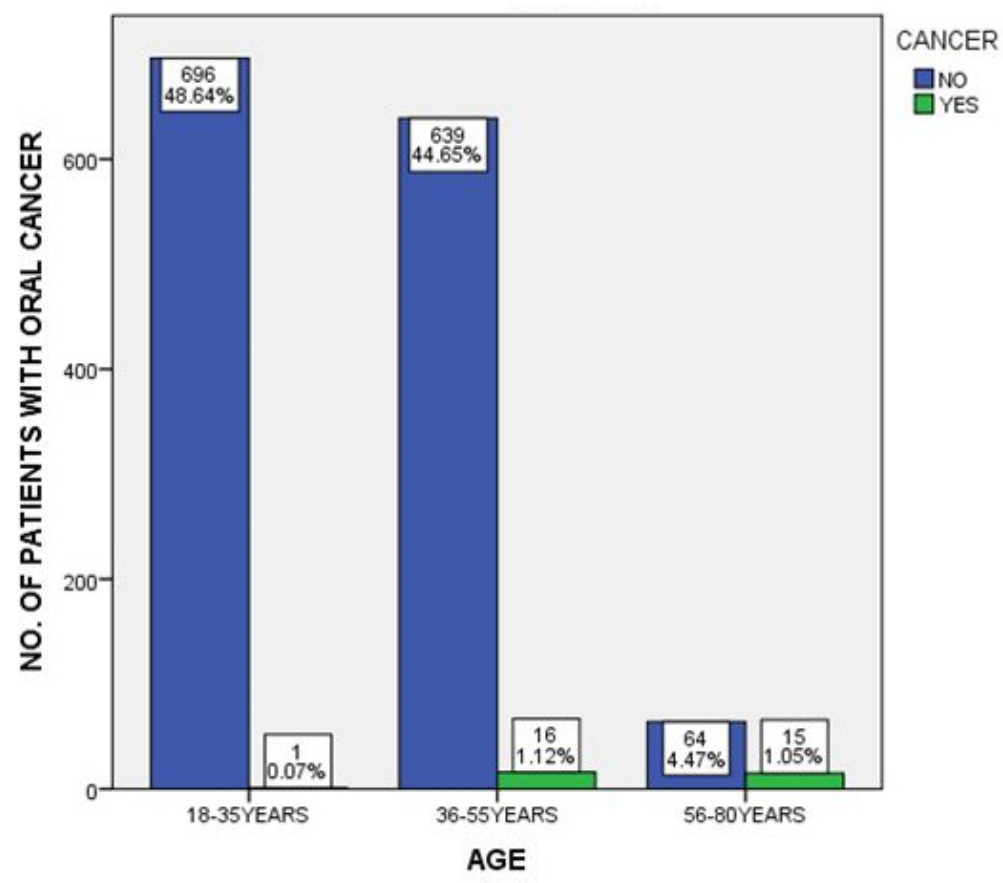

Figure 3: This is a bar graph showing an association between the age of the patients and oral cancer. The $\mathrm{X}$-axis denotes the age of the patients and Y-axis denotes the number of patients with oral cancer. Among the 32 patients with oral cancer, 16 patients were in the age group of 36-55 years (green) for which the P-value was found to be statistically significant $(<0.05)$. Thus there is a significant association between age and the occurrence of oral cancer. 